\title{
New Radiofrequency Mass Spectrometer Having High Duty Cycle*
}

\author{
Robert M. Mills
}

(Received December 11, 1962)

\begin{abstract}
A new type of time-of-flight mass spectrometer has been developed which, unlike most other such instruments, utilizes a large fraction of the nonpulsed stream of ions. The ions are accelerated through a d-c voltage and then pass into a drift tube. A radiofrequency voltage is applied at the exit and entrance gaps of the drift tube in such a way that the field at the entrance is the negative of that at the exit. The number of ions which pass through the drift tube with such a velocity that the energy change from the rf fields is a minimum, is measured by placing an electrical barrier in front of the collector and differentiating the cutoff curve. Velocity modulation of the beam resulting from the rf field at the first gap limits the instrument to moderate resolution. However, the instrument has a 60 percent duty cycle, a value significantly greater than that achieved by most other time-of-flight mass spectrometers. It also has the advantages of electrical and mechanical simplicity. Due to the nature of the instrument's output, it will be most useful in applications requiring the analysis of a simple mass spectrum and where high duty cycle is of real value. The instrument has resolved the isotopes of rubidium at masses 85 and 87 , and has identified one component ion beams ranging in mass from helium to cesium.
\end{abstract}

\section{Introduction}

This paper describes a new type of time-of-flight spectrometer which has a large duty cycle along with moderate resolving power. It was developed for use in flame research investigations at the $\mathrm{Na}$ tional Bureau of Standards. Two types of experiments have been planned. The first is that of measuring the appearance potential of certain halogenated compounds. In experiments of this type, one looks for the appearance of a single ion peak as the energy of the ionizing electron is increased. High sensitivity is important if the appearance potential is to be determined accurately. The second application is that of analyzing ions drawn directly from a flame. J Other laboratories that have used flames as the 10 source have experienced difficulty in drawing enough ions from the flame for analysis. The high duty cycle of the mass spectrometer described in this paper should make the instrument efficient in using the ions which are available in these two experiments.

Most of the previous efforts to increase the sensitivity of mass spectrometers have been directed at the collector end by using electron multipliers. An electron multiplier suffers from a changing gain over a period of time and was not used in the instrument described here. However, it would be possible to incorporate one if an even greater sensitivity were desired.

In general, time-of-flight (T.O.F.) mass spectrometers depend on the fact that, given equal energy, heavy ions take a longer time to traverse a given distance than do light ions. The velocity of any ion varies according to:

* A portion of the funds for support of the work reported was provided by the Bureau of Ships, Department of the Navy.

$$
v=\sqrt{\frac{2 q V}{m}}
$$

where $q$ is the charge of the ion, $V$ is the total voltage through which the ion has been accelerated, and $m$ is the mass of the ion. If a pulse of ions of various masses enters a field-free drift tube, after being accelerated, the ions will travel in bunches corresponding to their mass. 'The distance between bunches will increase the further the ions travel down the drift tube. The mass spectrum can then be measured by observing the ion current collected at the exit end of the drift tube as a function of time.

All instruments of this type have to be pulsed at the source and the time between pulses must be great enough to clear the drift tube of ions from the previous pulse. The pulsing greatly reduces the duty cycle of the mass spectrometer which thus lowers its time average collector current. The instrument of this type which is best known was reported by Wiley and McLaren in 1955 [1]. ${ }^{1}$ Ions are created by a $0.25 \mu$ sec electron pulse once every $100 \mu \mathrm{sec}$ [2], giving a duty cycle of 0.25 percent.

Another type of T.O.F. instrument which is described in the literature $[3,4]$ distinguishes masses by the energies the ions receive from a series of rf fields. A continuous beam of ions passes through 10 or more electrodes which are perpendicular to the beam path. The geometry of these electrodes and frequency of the rf fields maintained between them are such that ions of one mass receive more energy than lighter or heavier ions. An electrical barrier is placed in front of the ion collector. Only ions which have received extra energy from the rf fields are able to overcome the barrier and reach the collector. This instrument gives good resolution,

Figures in brackets indicate the literature references at the end of this paper 
but has several drawbacks. The spacing between the electrodes is critical for proper operation which makes construction and modification difficult. The numerous electrodes through which the ions must travel reduce the transmission of the instrument. And, finally, this type of instrument collects pulses of ions even though a continuous beam of ions enters the analyzing region. This effect is due to the fact that an ion of correct mass must also have the correct initial phase with respect to the rf voltages as it enters the fields in order to gain the necessary amount of energy to reach the collector. Thus the sensitivity of the instrument is reduced in the same way as with the pulsed type of spectrometer. The duty cycle is given in reference [4] as $\frac{N}{R 8 \sqrt{3}}$, where $N$ is the number of stages and $R$ is the resolution. An instrument of this type having 20 stages thus has a duty cycle less than 3 percent at a resolving power of 50 .

\section{Theory of Operation}

The mass spectrometer reported here resembles the last instrument described in that ions of different masses are distinguished by their energies rather than their spatial positions. It differs in that initial phase requirements are much less severe.

Consider a drift tube of length $L$ which has $\mathrm{rf}$ electric fields applied at its ends as shown in figure 1. Tungsten screens, not shown in the figure, are attached to the ends of the drift tube and define the entrance and exit. It is seen that the grids near each end of the drift tube are at the same potential. Therefore, at a given instant, the rf fields between the drift tube and the grids are exactly equal and opposite at the entrance and exit ends of the tube. These rf fields are represented by the upper and lower curves in figure 2.

The operation of the instrument can be explained most easily if the flight time of a given mass through the drift tube is assumed to be constant. This is not entirely a valid assumption since the effects of the variation in flight time (velocity modulation)

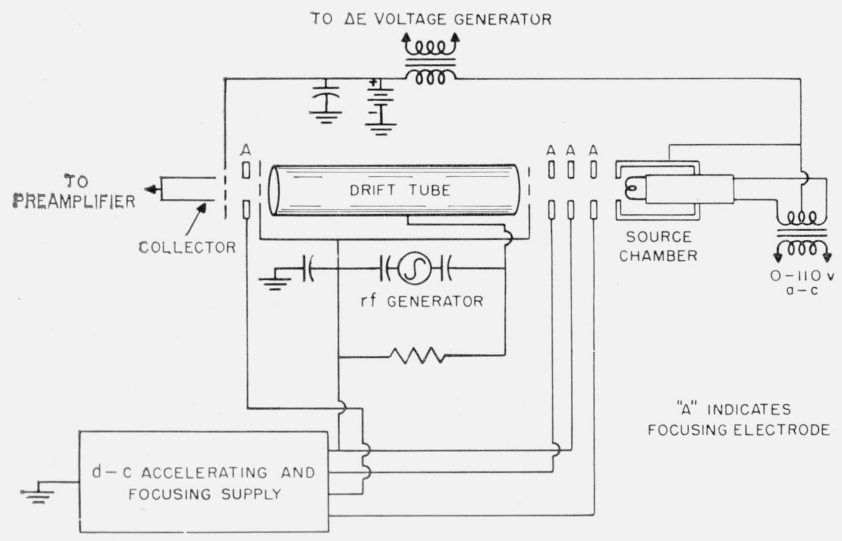

Figure 1. Simplified diagram of the mass spectrometer.

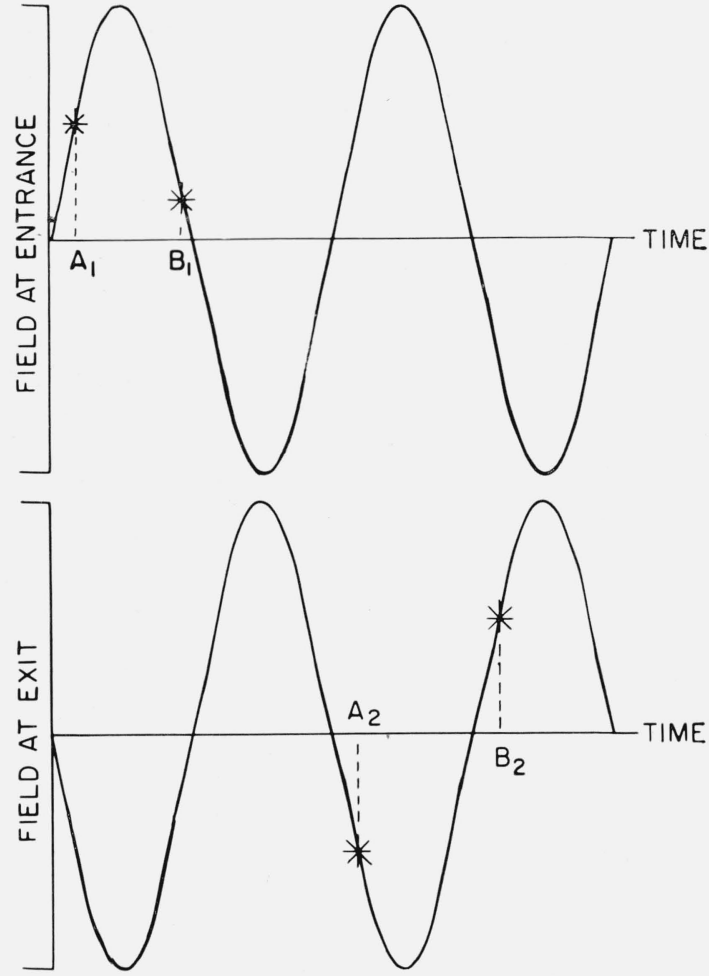

FIgure 2. Radiofrequency fields at the entrance and exit of drift tube.

A resonant ion entering at time $A_{1}$ and leaving at time $A_{2}$ experiences no change in energy. A typical nonresonant ion entering at time $B_{1}$ and leaving at time $B_{2}$ experiences a net gain in energy.

resulting from the rf voltage at the entrance of the drift tube are quite noticeable. Nevertheless, velocity modulation will be neglected until the resolution and duty cycle of the instrument are discussed.

Suppose an ion enters the drift tube at some time $A_{1}$. If the frequency of the rf voltage and the velocity of the ion are such that the ion emerges at the other end of the drift tube after one period of the rf wave, the ion will pass through the second rf field at time $A_{2}$. It is seen that no matter what part of the cycle the ion enters the drift tube, the energy which it obtains at the first field is exactly balanced out by the energy it loses at the second. Consider now, a heavier ion which takes a longer time to traverse the drift tube. For example, if it enters the drift tube at time $B_{1}$, it might pass through the exit field at time $B_{2}$. Any ion which does not pass through the drift tube in an integral number of cycles will undergo an increase or decrease in energy, depending upon when it entered the drift tube. A beam of such ions which is homogeneous in energy at the entrance of the drift tube will emerge with a distribution of energies. However, a similar stream of ions which passes through the tube in an integral number of rf cycles will emerge with all the ions unchanged in energy. These are the ions which the detection system measures. 


\section{Detection System}

As stated before, the detection system must identify the resonant ions by their energy. This measurement is made by the retarding field method $[5,6]$. An electrical barrier is placed immediately in front of the Faraday cage collector. For a given barrier potential, $E$, measured with respect to the ion source, only ions with energies equal to $E$ electron volts or greater can pass. Or

$$
S(E)=\int_{E}^{\infty} N(e) d e
$$

where $S(E)$ is the number of ions per unit of time that penetrate a barrier of height $E$ and $N(e)$ is the number of ions approaching the barrier per unit of time with energy $e$ at the barrier. $N(e)$ is obtained from (2) by differentiating with respect to $E$

$$
\frac{d S(E)}{d E}=-N(E)
$$

Since the detection system must measure the number of ions which have had their energies unchanged by the rf fields, and, since these particular ions arrive at the barrier with the same energy that they had at the source, $N(0)$ is the quantity sought. Figure 3 is a set of cutoff curves showing collector current as a function of barrier height in volts, obtained with an $x-y$ recorder. The center curve is the cutoff curve for a potassium beam which is in resonance with the rf voltage, taken with $n$ equal to 12 . Here, $n$ is equal to the number of rf cycles which an ion takes to traverse the drift tube. The other curves were taken with $n$ equal to $11.5,11.7,12.2$, and 12.4.

The differentiation represented in eq (3) is performed in the instrument by introducing a variation in $E$ about the point $E=0$ shown as $\Delta E$ in figure 3. The resulting a-c component in the collector current is proportional to $d S(E)$. And $d S(E)$ is proportional to $N(e)$ if $d E$ is infinitely small. A square wave of magnitude $\pm 3.9 \mathrm{v}$ was used to produce this variation and this value will be assumed in the remainder of the paper. Using a $d E$ of finite size means that an average value of $N$ is actually measured over the range $E=0 \pm 3.9$ v. If the ion beam is composed of more than one mass, the resulting a-c signal is the sum of the contributions from the individual masses.

The variation in the ion current was amplified by a-c amplifiers and then fed to a synchronous detector, $\Delta E$ supplying the reference phase used in the detector. An $x-y$ recorder was connected to the output of the detector. The masses were swept by changing the frequency of the rf voltage. A potentiometer was attached to the tuning knob of the rf generator. The voltage from the potentiometer was thus related to the rf frequency and was connected to the other input of the $x-y$ recorder. The potentiometer and tuning knob were driven by a $1 \mathrm{rpm}$ motor.

Figures $4,6,7 \mathrm{a}, 7 \mathrm{~b}$, and 8 were made using this

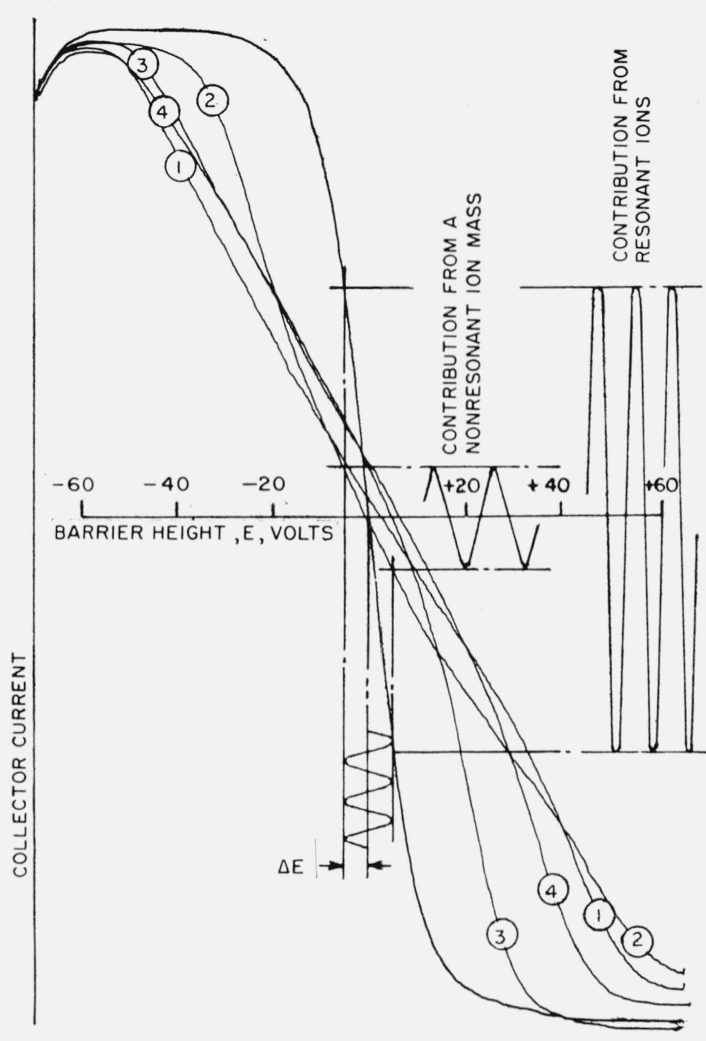

Figure 3. Cutoff curves obtained from an $\mathrm{x}-\mathrm{y}$ recorder for $\mathrm{n}=12.0$ (central curve) $\mathrm{n}=11.5(1), \mathrm{n}=11.7(2), \mathrm{n}=12.2(3)$, and $\mathrm{n}=12.4(4)$ showing the difference in output signals for resonant and nonresonant ions.

A square wave was used for $\Delta E$ instead of the sine wave shown.

arrangement. The abscissa is not linear in frequency. No development work has been done to make this instrument a fast-sweep instrument, capable of displaying its output on an oscilloscope, but this seems feasible.

The advantage of picking out the ions with zero energy change should be noted. Instruments of the type in reference [4] must maintain a constant ratio of barrier height to rf voltage to obtain the desired resolution. However, if the number of ions with zero energy change is measured instead of the number with maximum energy change, the strong dependence on resolution no longer exists and less care need be taken in controlling the rf amplitude as the rf frequency is changed.

\section{Harmonics}

As has been mentioned before, ions may stay in the drift tube any whole number of cycles in order to be detected. Thus, as the frequency is varied, the mass peaks will repeat corresponding to various values of $n$ as in figure 4 . The two rf frequencies at which adjacent harmonics of a particular ion appear must be measured to determine the ion's mass. The ratio of these two frequencies is equal to 


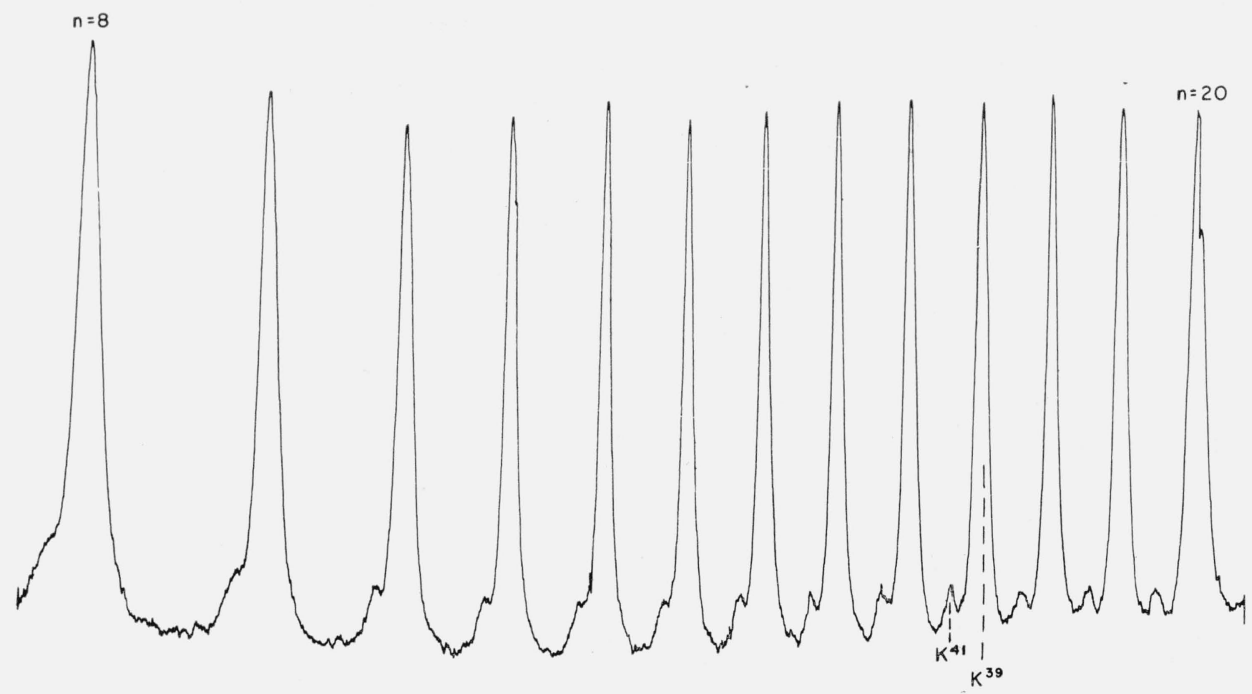

FiguRE 4. The a-c collector current as a function of rf frequency as plotted by an x-y recorder.

The eight through twentieth harmonies of $\mathrm{K}^{39}$ and $\mathrm{K}^{41}$ are shown.

the ratio of their harmonic numbers. That is:

$$
\frac{f_{n}}{f_{n+1}}=\frac{n}{n+1} \text {. }
$$

This equation can be solved for $n$, or $n$ can be found conveniently on a slide rule. The procedure is to look for two consecutive integers which have the same ratio as $f_{n} / f_{n+1}$. Knowing $n$ and the corresponding frequency, $f_{n}$ (equal to $1 / T_{n}$ ), the flight time of the ion, $t$, is found from $t=n T_{n}$. If the length of the drift tube is $L$, eq (1) can be solved for $m$, using $v=L / t$.

The determination of $m$ becomes difficult if the beam contains ions of many masses. The harmonics of the mass peaks become too cluttered to pick out two adjacent harmonics. In applications requiring the study of complex beams, it would be desirable to eliminate all but one of the harmonics for each mass. Partial elimination of the harmonics has been achieved by applying two radiofrequencies, referred to as $f_{l}$ and $f_{h}$, as in figure 5. Frequency $f_{l}$ is generated from $f_{h}$ by frequency division so that the ratio of $f_{h}$ to $f_{l}$ is a known whole number, say $r$.

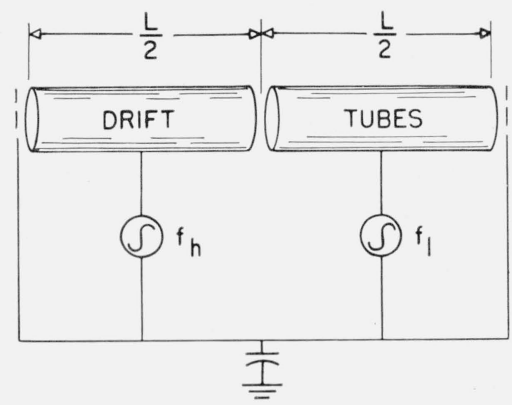

FIGURE 5. Drift tube arrangement used to eliminate harmonics.
With the present equipment, $r$ can be chosen to be equal to $2,4,8,16$, or 32 . In order for the ions to escape a change in energy, they must pass through two drift tubes emerging from each tube after an integral number of $\mathrm{rf}$ cycles. Let $n_{l}$ be the number of rf cycles an ion takes to travel through the first drift tube, and $n_{h}$ the number of rf cycles an ion takes to travel through the other tube. Since both tubes are of equal length, the transit times for both tubes must be equal. That is, $n_{l} T_{l}=n_{h} T_{h}$. By definition, $f_{l}=1 / T_{l}$ and $f_{h}=1 / T_{h}$.

Then

$$
n_{l}=n_{h} \frac{T_{h}}{T_{l}}=\frac{n_{h}}{r} .
$$

If $n_{l}$ is equal to one, $n_{h}$ must be equal to $r$. If $n_{l}$ is equal to any integer, $k, n_{k}$ must be equal to $k r$. In practice the possibility of $k$ being greater than one for a particular mass is eliminated by sweeping over an rf frequency range of less than one to two. Thus, an ion which would pass through the second drift tube with $n_{h} \neq r$ does not pass through the first drift tube in a whole number of rf cycles. The voltage at frequency $f_{l}$ discriminates against the unwanted harmonics while the voltage at frequency $f_{h}$ resolves the masses as before. As is the case in using a single drift tube, each rf voltage can be increased until the improvement in resolution is balanced out by the adverse effects of velocity modulation.

Figure 6 was obtained by using this principle of harmonic elimination. It shows the frequency range where the 10th through 28th harmonics of potassium would normally appear. The largest peak at the center is the 16 th harmonic of $\mathrm{K}^{39}$. The two small peaks on either side are the 15 th and 17th harmonics of $\mathrm{K}^{39}$. Figure 6 was made using the maximum low frequency voltage which the equipment could generate. 


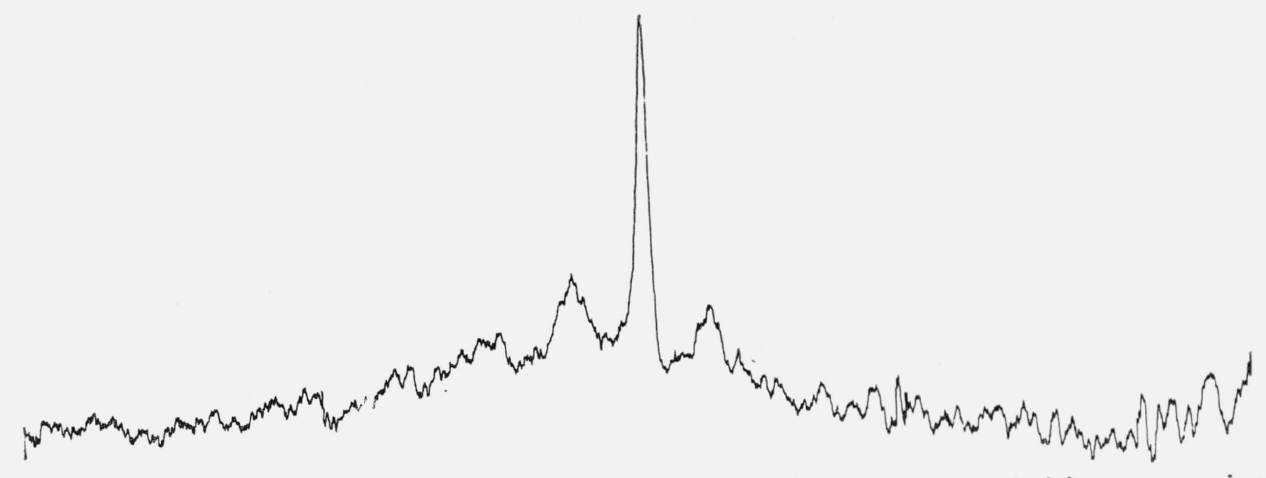

Figure 6. An x-y recorder plot of the a-c collector current as a function of $r f$ frequency using the harmonic elimination equipment.

The large peak is the 16 th harmonic of $\mathrm{K}^{39}$. The smaller peaks on either side are the 15 th and 17 th harmonic of $\mathrm{K}^{39}$
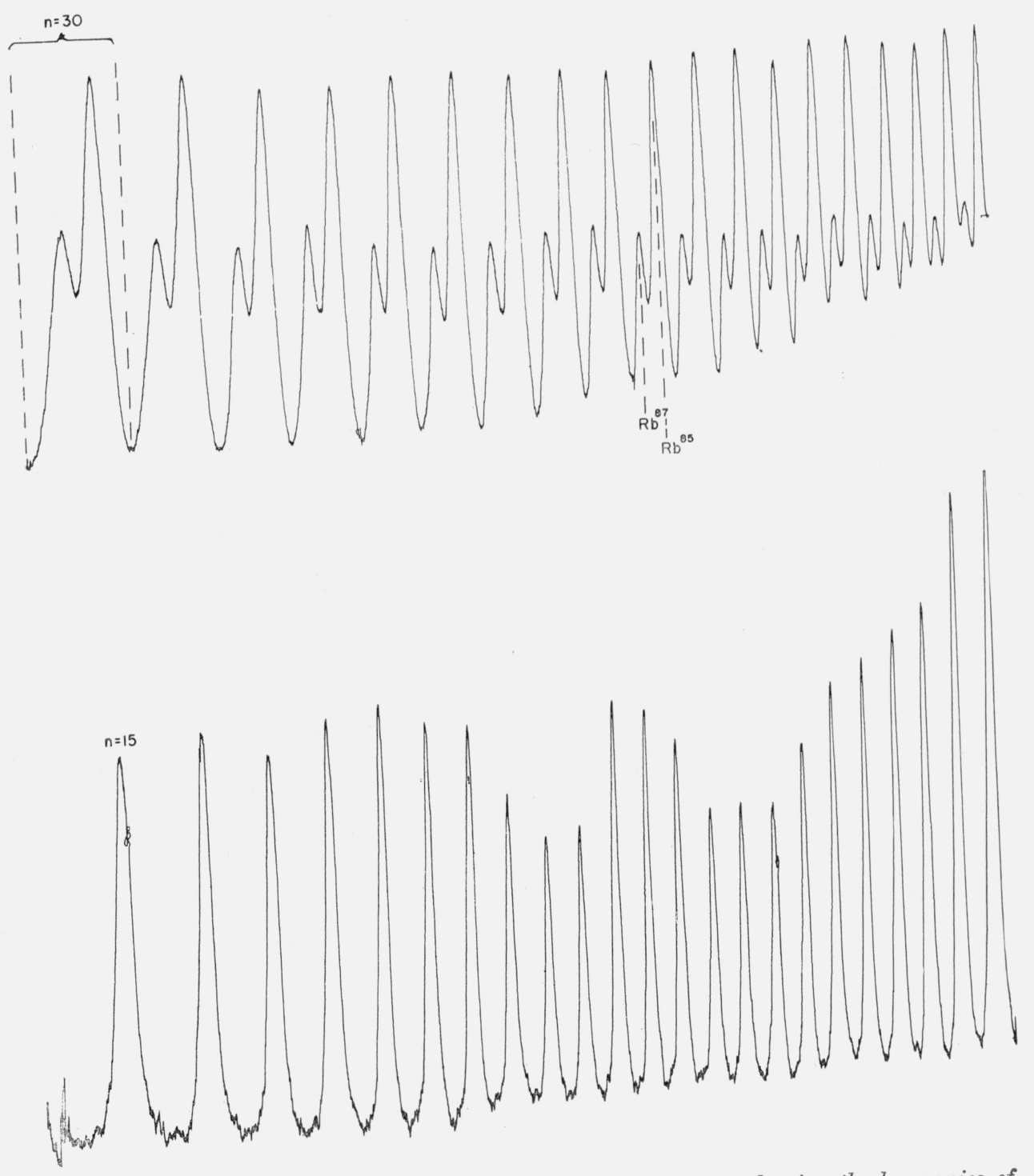

FIGURE 7. The a-c collector current as a function of $r f$ frequency showing the harmonics of $\mathrm{Rb}^{85}$ and $\mathrm{Rb}^{87}(a)$ and of cesium $(b)$.

The plots were obtained by using an $x-y$ recorder. 


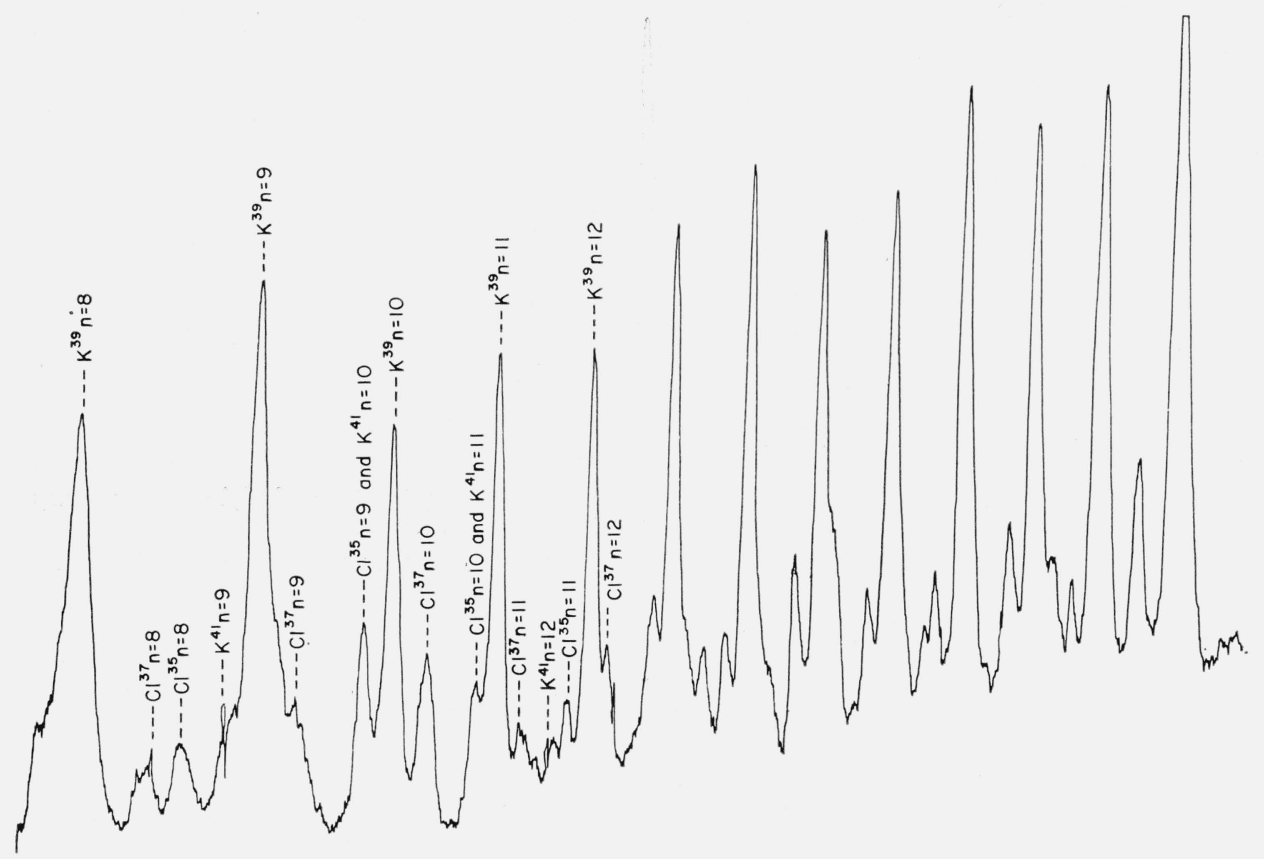

FIGURE 8. An x-y recorder plot showing a-c collector current as a function of $r f$ frequency. Shown are harmonics of $\mathrm{K}^{39}, \mathrm{~K}^{41}, \mathrm{Cl}^{35}$ and $\mathrm{Cl}^{37}$.

\section{Resolution and Duty Cycle}

In order to find the resolution and duty cycle which can be expected from this instrument (without harmonic elimination), the effect of the rf voltage at the entrance of the drift tube on the flight time of the ion must be considered. Neglecting initial energy, the energy of an ion after passing through both rf gaps can be expressed as:

$$
e=q\left\{V_{\mathrm{dc}}+V_{\mathrm{rf}} F[\sin \omega \tau-\sin \omega(\tau+t)]\right\}
$$

where

$V_{\text {dc }}$ is the d-c accelerating voltage,

$V_{\mathrm{r} f}$ is the amplitude of the radiofrequency voltage, $t$ is the time of flight of the ion through the drift tube, $\tau$ is the time at which the ion enters the drift tube, $\omega$ is the angular frequency of the rf voltage,

$F$ is the transit-time factor due to the fact that the gap widths are of finite size.

$F$ can be shown to be very nearly equal to unity by a calculation similar to that in reference [7]. Under the conditions that the gap width is $2 \mathrm{~mm}, L$ is $1 \mathrm{~m}, n=20$, and that $V_{\mathrm{rf}}<<V_{\mathrm{dc}}, F$ is found to be 0.997 . Since the above conditions are valid approximations for this instrument, $F$ will be assumed to be unity.

By writing $v=\frac{L}{t}$ in eq $(1)$ :

$$
t=t_{0}\left[1+\frac{V_{\mathrm{rf}}}{V_{\mathrm{dc}}} \sin \omega \tau\right]^{-\frac{1}{2}}
$$

where $t_{0}$ is the flight time of an ion which enters the drift tube when the rf voltage is zero and is equal to

$$
t_{0}=L \sqrt{\frac{m}{2 q V_{\mathrm{dc}}}} .
$$

Substituting (7) in (6), the net energy in electron volts which the ion receives from the rf fields is seen to be:

$e_{\mathrm{rf}}=V_{\mathrm{rf}}\left\{\sin \omega \tau-\sin \left[\omega \tau+\omega t_{0}\left(1+\frac{V_{\mathrm{rf}}}{V_{\mathrm{dc}}} \sin \omega \tau\right)^{-\frac{1}{2}}\right]\right\}$

Thus $e_{\text {re }}$ is not zero under the condition that $t_{0}$ is a whole number of cycles as was assumed to be the case when velocity modulation was neglected.

If the magnitude of $e_{\mathrm{rf}}$ is larger than $3.9 \mathrm{ev}$, the ion will not be represented in the collector output signal. The theoretical peak shape was found by plotting $e_{\text {rf }}$ as a function of $\omega \tau$ between zero and $2 \pi$ for various $\omega t_{0}$. Then for each value of $\omega t_{0}$ the fraction of the cycle over which $-3.9<e_{\mathrm{rf}}<+3.9$ ev was measured, and this fraction was plotted as a function of $\frac{\omega t_{0}}{2 \pi}$ in figure 9 over the range 19.5 $<\frac{\omega t_{0}}{2 \pi}<20.5$. Values of $V_{\mathrm{rr}}$ and $V_{\mathrm{dc}}$ used were the measured values when $t_{0}$ equaled 20 times $T$ for potassium $\left(\mathrm{K}^{39}\right)$ : 24 volts and 3000 volts, respectively. It is seen in figure 9 that as a result of velocity modulation (a) the maximum duty cycle does not actually occur when $\frac{\omega t_{0}}{2 \pi}=\frac{t_{0}}{T}$ is a whole number as was 
the case when velocity modulation was neglected, and (b) the duty cycle at its maximum is equal to 61 percent.

The duty cycle actually obtained under the above conditions was measured by presetting the rf frequency for maximum collector output signal. The ratio of the a-c collector current with rf voltage on to that with rf voltage off was found to be about 60 percent.

For a given radiofrequency, the abscissa of figure 9 may be converted to mass numbers by eq (8). With $\frac{\omega t_{0}}{2 \pi}$ equal to 20 for mass number $39, \frac{\omega t_{0}}{2 \pi}=20.5$ corresponds to mass number 41.

Up to a point, the difference in $e_{\mathrm{r} f}$ between two ions slightly different in mass increases as the time rate of change of the $\mathrm{rf}$ voltage increases. The time rate of change can be increased by increasing the rf frequency or by increasing the magnitude of the rf voltage. However, increasing the rf voltage increases the amount of velocity modulation, and it can be seen in figure 9 that velocity modulation causes a broadening in the peak shape. Increasing the frequency of the rf voltage increases the adverse effect of whatever velocity modulation is present. It is thus reasonable to assume that there exist values of $V_{\text {rf }}$ and $\omega$ which give a maximum resolution.

An analysis of the peak shape was made only for the values of $V_{\text {rf }}$ and $\omega t_{0}$ given above. These values were chosen because they produced good resolution between $K^{39}$ and $K^{41}$ (see fig. 4). It is possible that a further study might show that other values would result in better resolution.

It is assumed in figure 9 that (a) the ions are monoenergetic at the source, (b) they do not lose energy by collisons as they travel through the instrument, (c) they are in perfect focus at the barrier [7], and (d) ions with insufficient energy are cut off with perfect sharpness at the barrier. ${ }^{2}$ The center curve in figure 3 indicates a final energy spread in the order of 30 electron volts, probably due mainly to the failures of assumption (a) and (d). Failure of any of the above assumptions results in a lower current efficiency, as well as a broadening of the peak shape. A careful study of these factors has not been made.

\section{Results}

The following positive ions have been detected with this instrument: $\mathbf{K}^{39}, \mathrm{~K}^{41}, \mathrm{Na}^{23}, \mathrm{He}^{4}, \mathrm{~N}_{2}^{+}, \mathrm{N}^{++}$, $\mathrm{Cl}^{35}, \mathrm{Cl}^{37}, \mathrm{Rb}^{85}, \mathrm{Rb}^{87}$, and $\mathrm{Cs}^{133}$. All except the helium and nitrogen ions were obtained by placing salts on a hot tungsten wire filament [9]. Helium and nitrogen were ionized by heating a bare tungsten filament in the respective atmospheres at reduced pressures.

The d-c accelerating voltage was maintained at approximately $3,000 \mathrm{v}$, and the drift tube was roughly $1.12 \mathrm{~m}$ long $(0.56 \mathrm{~m}$ long when used with the harmonic eliminator). In order to avoid the necessity of measuring $V$ and $L$ accurately, a calibration

\footnotetext{
2 For sharp cutoff, the barrier must be such that no field can penetrate through ${ }^{2}$ For sharp cutoff, the barrier must be such that no field can penetrate through
it. The collector must be able to collect ions of large and small energy with equal it. The collector must be able to collect ions of large and small energy with equal
efficiency and be able to prevent ions which have experienced elastic reflections from escaping the collector.
}

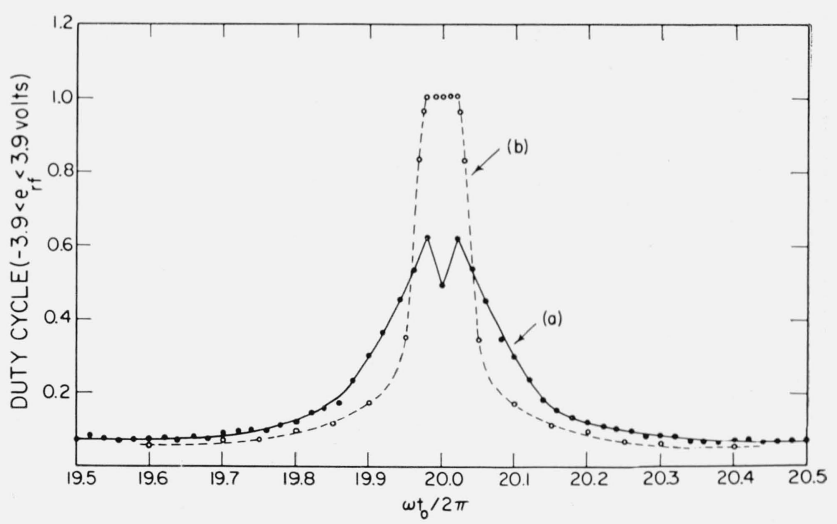

Figure 9. Computed peak shape, (a) with velocity modulation, and $(b)$ when velocity modulation is neglected.

If $\omega$ is set so that $t_{\circ}=20 T$ for $\mathrm{K}^{39}, \omega t_{\circ} / 2 \pi=20.5$ corresponds to $\mathrm{K}^{41}$.

actor $\mathrm{d}$ efined as $\mathrm{CF}=\frac{1}{T_{n}} \frac{\sqrt{m}}{n}=\frac{\sqrt{2 e V}}{L}$ was measured for each of the ions listed above. It was found to be equal to $0.678 \pm 0.003(\sim 0.4 \%)$ if $m$ is expressed in atomic mass units $\left(\mathrm{O}^{16}=16.00\right)$ and $1 / T$ in megacycles.

This figure was obtained by using known values of mass for the above ions. The frequencies at which the various harmonics appeared were measured with a timer-counter. The percent fluctuation of the calibration factor is an indication of the precision of which the instrument is capable. With the present source, it is necessary to apply a focusing field in the region where the filament is located. The proper focusing voltage changes each time the filament is removed to replace the salt. It has been found that this change in focusing voltage produces a slight change in the calibration factor, accounting for a portion of the 0.4 percent variation. For a fixed value of the focusing voltage, the calibration factor is found to vary about 0.1 percent. Figure 7 a shows the 30th through 48th harmonics of $\mathrm{Rb}^{85}$ and $\mathrm{Rb}^{87}$; figure $7 \mathrm{~b}$ shows the 15 th through 37 th harmonics of $\mathrm{Cs}^{133}$. Figure 8 is a mass spectrum showing $\mathrm{K}^{39}, \mathrm{~K}^{41}, \mathrm{Cl}^{35}$, and $\mathrm{Cl}^{37}$. Some variation in the peak heights of the harmonics can be observed in figures $7 \mathrm{~b}$ and 8 . Duplicating these graphs shows that this fluctuation is due to the instability of the source. Little care has been taken, up to date, to ensure linearity in peak heights.

In figure 10 , the ion source at the right, the tube containing the drift tube, the collector shielding box, and some of the electronic equipment are visible.

Because of the cluttering of the harmonic peaks, this instrument will be useful in experiments requiring the analysis of ion beams containing a small number of mass components such as those mentioned in the introduction. In experiments where the cluttering is not a serious limitation, the instrument offers important advantages. In addition to the high duty cycle, it has light weight, and electrical and mechanical simplicity. There are no critical alinements or spacings. 


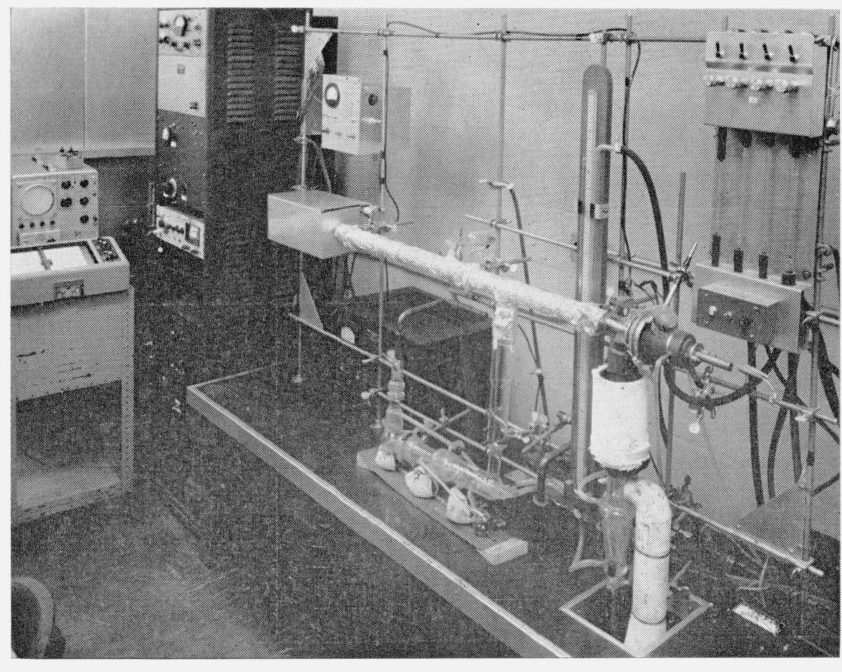

Figure 10. The mass spectrometer.

The author thanks E. Carroll Creitz for his help and interest, William H. Bailey for his shop work, Louis A. Marzetta and Malcom S. Morse for their work in developing the rf generators, Kenneth McCulloh for pointing out the importance of velocity modulation, and others who have made helpful suggestions.

\section{References}

[1] W. C. Wiley and I. H. MeLaren, Time-of-flight mass spectrometer with improved resolution, Rev. Sci. Instr. 26, 1150 (1955).

[2] D. B. Harrington, Advances in mass spectrometry, Edited by J. D. Waldron (Pergamon Press, New York, 1959).

[3] P. A. Redhead, A linear radio frequency mass spectrometer, Can. J. Phys. 30, 1 (1952).

[4] P.A. Redhead and C. R. Crowell, Analysis of the linear r. f. mass spectrometer, J. Appl. Phys. 24, 331 (1953).

[5] R. E. Fox, W. M. Hickam, D. J. Grove, and T. Kjeldass, Jr., Rev. Sci. Instr. 26, 1101 (1955).

[6] L. B. Leder and J. A. Simpson, Rev. Sci. Instr. 29, 571 (1958).

[7] J. J. Livingood, Principles of cyclic particle accelerators, p. 269 (D. Van Nostrand Co., Inc., Princeton, N.J., 1961).

[8] J. A. Simpson, Rev. Sci. Instr. 32, 1283 (1961).

[9] Mark G. Inghram and Richard J Hayden, Mass spectroscopy, Nuclear Science Series Report No. 14, National Academy of Sciences-National Research Council, Washington, D.C. (1954).

(Paper 67C4-139 